\title{
Symmetric and Positive Definite Broyden Update for Unconstrained Optimization
}

\author{
Saad Shakir Mahmood
}

Received 5/6/2018, Accepted 20/2/2019, Published 1/9/2019

\author{
Noor Salah Muhanah
}

\begin{abstract}
:
Broyden update is one of the one-rank updates which solves the unconstrained optimization problem but this update does not guarantee the positive definite and the symmetric property of Hessian matrix.

In this paper the guarantee of positive definite and symmetric property for the Hessian matrix will be established by updating the vector $y_{k}$ which represents the difference between the next gradient and the current gradient of the objective function assumed to be twice continuous and differentiable .Numerical results are reported to compare the proposed method with the Broyden method under standard problems.
\end{abstract}

Key words: Broyden update, Hessian matrix, Quasi Newton condition .

\section{Introduction:}

Many methods of the unconstrained optimization generate a Hessian matrix satisfying each of the principal properties of this matrix (positive definite and symmetric property). One of these methods is Boyden method, where the Hessian matrix is given in the following form

$\mathrm{B}_{\mathrm{k}+1}=\mathrm{B}_{\mathrm{k}}+\frac{\left(\mathrm{y}_{\mathrm{k}}-\mathrm{B}_{\mathrm{k}} \mathrm{s}_{\mathrm{k}}\right)_{\mathrm{k}}^{\mathrm{T}}}{\left\|\mathrm{s}_{\mathrm{k}}\right\|^{2}}$

Where

$\mathrm{B}_{\mathrm{k}+1}$ is the next approximation of Hessian matrix, $\mathrm{B}_{\mathrm{k}}$ is the current approximation of Hessian matrix , $\mathrm{y}_{\mathrm{k}}=\mathrm{F}\left(\mathrm{x}_{\mathrm{k}+1}\right)-\mathrm{F}\left(\mathrm{x}_{\mathrm{k}}\right)[5]$,

$s_{\mathrm{k}}=\mathrm{x}_{\mathrm{k}+1}-\mathrm{x}_{\mathrm{k}}[5]$,

$\mathrm{f}: \mathrm{R}^{\mathrm{n}} \rightarrow$

$\mathrm{R}$ is continuous and differentiable function

$\mathrm{x}_{\mathrm{k}+1}$ is the next sollution,

$\mathrm{x}_{\mathrm{k}}$ is the current solution. $\mathrm{F}\left(\mathrm{x}_{\mathrm{k}}\right)$ be the gradient of $f$ at $x_{k}$.

The positive definite property is very important to guarantee the existence of the minimizer of the objective function, because the Hessian matrix is symmetric ( $f$ is continuous), so the symmetric property of matrix $B_{k}$ is very important to guarantee the convergence of $B_{k+1}$ to the orginal

Hessian matrix .

Symmetric Positive Definite Broyden Rank One Update (SPBR1 update)

Given $\mathrm{f}: \mathrm{D} \subseteq \mathrm{R}^{\mathrm{n}} \rightarrow \mathrm{R}$ f is assummed to be continuous and differentiable on $\mathrm{D}$, Based on

Zhang Xu condition

Department of Mathematics, College of Education, Al Mustansiriyah University, Baghdad, Iraq

*Corresponding author: $\mathrm{y}_{\mathrm{k}}^{*}=\lambda_{k} \mathrm{y}_{\mathrm{k}}$

where $\lambda_{k}$ is a matrix can be determined to guarantee the symmetric and positive

definite properties of $B_{k+1}$, from the Broyden update and (4)

$\mathrm{B}_{\mathrm{k}+1}=\mathrm{B}_{\mathrm{k}}+\frac{\left(\mathrm{y}_{\mathrm{k}}^{*}-\mathrm{B}_{\mathrm{k}} \mathrm{s}_{\mathrm{k}}\right) \mathrm{s}_{\mathrm{k}}^{\mathrm{T}}}{\mathrm{s}_{\mathrm{k}}^{\mathrm{T}} \mathrm{s}_{\mathrm{k}}}$

Now to guarantee the symmetric property of the next approximation of Hessian matrix, suppose that $\mathrm{s}_{\mathrm{k}}=\mathrm{y}_{\mathrm{k}}^{*}-\mathrm{B}_{\mathrm{k}} \mathrm{s}_{\mathrm{k}} \quad \ldots$ (6)

By substituting (6)in (5) we get $B_{k+1}=B_{k}+$ $\frac{s_{k} s_{k}{ }^{T}}{s_{k}{ }^{T} s_{k}} \quad \ldots(7)$

From (6) $\lambda_{k} \mathrm{y}_{\mathrm{k}}=\mathrm{B}_{\mathrm{k}} \mathrm{s}_{\mathrm{k}}+\mathrm{s}_{\mathrm{k}} \quad \ldots$ (8)

The solution of the above equation for $\alpha_{k}$ is

$\lambda_{k}=\frac{\left(\mathrm{B}_{\mathrm{k}} \mathrm{s}_{\mathrm{k}}+\mathrm{s}_{\mathrm{k}}\right) \mathrm{y}_{\mathrm{k}}^{\mathrm{T}}}{\mathrm{y}_{\mathrm{k}}^{\mathrm{T}} \mathrm{y}_{\mathrm{k}}} \in \mathrm{R}^{\mathrm{nxn}} \quad \ldots$

And

$B_{k+1(\text { SPBR } 1)}=B_{k}+\frac{\left(\left(\frac{\left(B_{k} s_{k}+s_{k}\right) y_{k}^{T}}{y_{k}^{T} y_{k}}\right) y_{k}-B_{k} s_{k}\right) s_{k}{ }^{T}}{s_{k}^{T} s_{k}}$ ...(10)

\section{Theorem (1)}

The Hessian matrix $B_{k+1}$ in SPBR1 update satisfies the extended Q-N condition. (Zhang xu condition [7]) $\mathrm{B}_{\mathrm{k}+1} \mathrm{~s}_{\mathrm{k}}=\mathrm{y}_{\mathrm{k}}^{*}$

Proof:

$$
\begin{aligned}
B_{k+1} s_{k} & =\left(B_{k}+\frac{s_{k} s_{k}^{T}}{s_{k}^{T} s_{k}}\right) s_{k} \\
& =B_{k} s_{k}+\frac{s_{k} s_{k}^{T}}{s_{k}^{T} s_{k}} s_{k} \\
& =B_{k} s_{k}+s_{k}
\end{aligned}
$$

By equation (6) the proof is completed . 


\section{Theorem (2)}

The Hessian matrix $B_{k+1}$ produced by SPBR1 update $B_{k+1(S P B R 1)}=B_{k}+\frac{s_{k} s_{k}^{T}}{s_{k}^{T} s_{k}}$ is symmetric.

Proof:

$$
\begin{aligned}
& \mathrm{B}_{\mathrm{k}+1 \text { (SPBR1) }}=\mathrm{B}_{\mathrm{k}}+\frac{\mathrm{s}_{\mathrm{k}} \mathrm{s}_{\mathrm{k}}^{\mathrm{T}}}{\mathrm{s}_{\mathrm{k}}^{\mathrm{T}} \mathrm{s}_{\mathrm{k}}} \\
& \mathrm{B}_{\mathrm{k}+1 \text { (SPBR1) }}^{\mathrm{T}}=\mathrm{B}_{\mathrm{k}}^{\mathrm{T}}+\frac{\mathrm{s}_{\mathrm{k}}^{T^{T}} \mathrm{~s}_{\mathrm{k}}^{\mathrm{T}}}{\mathrm{s}_{\mathrm{k}}^{\mathrm{T}} \mathrm{s}_{\mathrm{k}}}
\end{aligned}
$$

Since $B_{k}^{T}=B_{k}$ and $s_{k}^{T^{T}}=s_{k}$, we obtain

$\mathrm{B}_{\mathrm{k}+1 \text { (SPBR1) }}^{\mathrm{T}}=\mathrm{B}_{\mathrm{k}+1 \text { (SPBR1) }}$

\section{Theorem (3)}

The

Hessian matrix produced by SPBR1 update is positively definite .

Proof:

By taking the equation (5)

let $0 \neq \mathrm{z} \in R^{n}$, then

$\mathrm{z}^{\mathrm{T}} \mathrm{B}_{\mathrm{k}+1} \mathrm{z}=\mathrm{z}^{\mathrm{T}}\left(\mathrm{B}_{\mathrm{k}}+\frac{\left(\mathrm{y}_{\mathrm{k}}^{*}-\mathrm{B}_{\mathrm{k}} \mathrm{s}_{\mathrm{k}}\right) \mathrm{s}_{\mathrm{k}}^{\mathrm{T}}}{\mathrm{s}_{\mathrm{k}}^{\mathrm{T}} \mathrm{s}_{\mathrm{k}}}\right) \mathrm{z}$

$z^{\mathrm{T}} \mathrm{B}_{\mathrm{k}+1} \mathrm{z}=\mathrm{z}^{\mathrm{T}} \mathrm{B}_{\mathrm{k}} \mathrm{z}+\frac{\mathrm{z}^{\mathrm{T}}\left(\mathrm{y}_{\mathrm{k}}^{*}-\mathrm{B}_{\mathrm{k}} \mathrm{S}_{\mathrm{k}}\right) \mathrm{s}_{\mathrm{k}} \mathrm{T}_{\mathrm{z}}}{\mathrm{s}_{\mathrm{k}}{ }^{\mathrm{T}} \mathrm{s}_{\mathrm{k}}} \quad \ldots$

By substituting (6) in (11) we get

$$
\begin{array}{r}
z^{T} B_{k+1} z=z^{T} B_{k} z+\frac{z^{T} s_{k} s_{k}^{T} z}{s_{k}^{T} s_{k}} \\
z^{T} B_{k+1} z=z^{T} B_{k} z+\frac{\left(s_{k}^{T} z\right)^{T}\left(s_{k}^{T} z\right)}{s_{k}^{T} s_{k}} \\
=z^{T} B_{k} z+\frac{\left\|s_{k}^{T} z\right\|^{2}}{\left\|s_{k}\right\|^{2}}
\end{array}
$$

Since $B_{k}$ is positive definite then $z_{k}^{T} B_{k} z_{k}>$

$0,\left\|s_{k}^{T} z\right\|$ and $\left\|s_{k}\right\|$ is always positive .Therefore $\mathrm{z}^{\mathrm{T}} \mathrm{B}_{\mathrm{k}+1} \mathrm{z}>0$.

\section{Local Linear Convergence of SPBR1}

In this section, the local linear convergence of SPBR1 update will be proved.

Consider the formula (10), we need the following assumptions:

\section{Assumption 1, [6]}

(A) $\quad \mathrm{f}: \mathrm{R}^{\mathrm{n}} \rightarrow \mathrm{R}$ is twice continuously differentiable on convex set $\mathrm{D} \subset \mathrm{R}^{\mathrm{n}}$.

(B) $\quad f(x)$ is uniformly convex, i.e., $\exists$ a positive constants $\mathrm{C}$ and $\mathrm{C}$

$\exists \forall \mathrm{x} \in \mathrm{L}(\mathrm{x})=\left\{\mathrm{x} \mid \mathrm{f}(\mathrm{x}) \leq \mathrm{f}\left(\mathrm{x}_{0}\right)\right\}$, we have:

$\mathrm{c}\|\mathrm{v}\|^{2} \leq \mathrm{v}^{\mathrm{T}} \nabla^{2} \mathrm{f}(\mathrm{x}) \mathrm{v} \leq \mathrm{C}\|\mathrm{v}\|^{2}$

for all $v \in \mathrm{R}^{\mathrm{n}}, c, \mathrm{C} \in \mathrm{R}^{+}$, $\mathrm{x}_{0}$ is starting point.

The assumption 1.B implies that $\nabla^{2} \mathrm{f}(\mathrm{x})$ is positively definite on $\mathrm{L}(\mathrm{x})$, and that $\mathrm{f}$ has a unique minimizer $\mathrm{x}^{*}$ in $\mathrm{L}(\mathrm{x})$.

By definition of positive definite and since equation (1), satisfy Zhang Xu condition.

then $\mathrm{V} \lambda_{k} \mathrm{y}_{\mathrm{k}}=\mathrm{s}_{\mathrm{k}}$ and $\lambda_{k} \mathrm{y}_{\mathrm{k}}=\mathrm{V}^{-1} \mathrm{~s}_{\mathrm{k}}$ then

$$
\lambda_{k} \mathrm{y}_{\mathrm{k}}=\widetilde{\mathrm{G}_{\mathrm{k}}} \mathrm{s}_{\mathrm{k}}
$$

where $\widetilde{\mathrm{G}_{\mathrm{k}}}=\mathrm{V}^{-1}$.
Now by equations (11), (7) and from definition of positive definite we get:

$\mathrm{C} \leq \frac{\left(\frac{\left(\mathrm{B}_{\mathrm{k}} s_{\mathrm{k}}+s_{\mathrm{k}}\right) \mathrm{y}_{\mathrm{k}}^{\mathrm{T}}}{\mathrm{y}_{\mathrm{k}}^{\mathrm{T}} \mathrm{y}_{\mathrm{k}}}\right) \mathrm{y}_{\mathrm{k}}^{\mathrm{T}} s_{\mathrm{k}}}{\left\|s_{\mathrm{k}}\right\|^{2}}=\frac{s_{\mathrm{k}}^{\mathrm{T}} \widetilde{\mathrm{G}_{k}} s_{\mathrm{k}}}{\left\|s_{\mathrm{k}}\right\|^{2}} \leq \mathrm{C}$

Where $\widetilde{\mathrm{G}_{\mathrm{k}}}$ is the average Hessian matrix, which is defined as

$\widetilde{\mathrm{G}_{\mathrm{k}}}=\int_{0}^{1} \nabla^{2} \mathrm{f}\left(\mathrm{x}_{\mathrm{k}}+\mu \mathrm{s}_{\mathrm{k}}\right) \mathrm{d} \mu$
and $\frac{1}{\mathrm{C}} \leq \frac{\left\|s_{\mathrm{k}}\right\|^{2}}{\left(\left(\frac{\left.\mathrm{B}_{\mathrm{k}} \mathrm{s}_{\mathrm{k}}+\mathrm{s}_{\mathrm{k}}\right) \mathrm{y}_{\mathrm{k}}^{\mathrm{T}}}{\mathrm{y}_{\mathrm{k}}^{\mathrm{T}} \mathrm{y}_{\mathrm{k}}}\right) \mathrm{y}_{\mathrm{k}}\right)^{\mathrm{T}} s_{\mathrm{k}}} \leq \frac{1}{\mathrm{c}}$

and also

$$
\begin{array}{r}
\frac{\left\|\left(\frac{\left(B_{k} s_{k}+s_{k}\right) y_{k}^{T}}{y_{k}^{T} y_{k}}\right) y_{k}\right\|^{2}}{s_{k}^{T}\left(\frac{\left(B_{k} s_{k}+s_{k}\right) y_{k}^{T}}{y_{k}^{T} y_{k}}\right) y_{k}}=\frac{\left\|\widetilde{G_{k}} s_{k}\right\|^{2}}{s_{k}^{T}\left(\frac{\left(B_{k} s_{k}+s_{k}\right) y_{k}^{T}}{y_{k}^{T} y_{k}}\right) y_{k}} \\
=\frac{\left.\left(\widetilde{G_{k}} s_{k}\right)^{T} \widetilde{\left(G_{k}\right.} s_{k}\right)}{s_{k}^{T} \widetilde{G_{k}} s_{k}}=\frac{s_{k}^{T} \widetilde{G_{k}} s_{k}}{s_{k}^{T} \widetilde{G_{k}} s_{k}}
\end{array}
$$

Assumption 1.B, means that $\widetilde{\mathrm{G}_{\mathrm{k}}}$ is positively definite, thus its square root is well defined. This is a symmetric square root ${\widetilde{\mathrm{G}_{\mathrm{k}}}}^{\frac{1}{2}}$ which satisfies $\widetilde{\mathrm{G}_{\mathrm{k}}}={\widetilde{\mathrm{G}_{\mathrm{k}}}}^{\frac{1}{2}} \cdot \widetilde{\mathrm{G}}_{\mathrm{k}}^{\frac{1}{2}}$

If we let $\mathrm{v}_{\mathrm{k}}={\widetilde{\mathrm{G}_{\mathrm{k}}}}^{\frac{1}{2}} \mathrm{~s}_{\mathrm{k}}$

then,

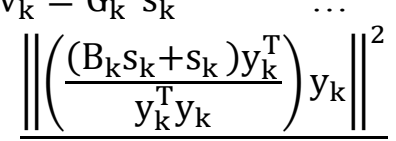

$=\frac{\left({\widetilde{\mathrm{G}_{\mathrm{k}}}}^{\frac{1}{2}} \mathrm{~s}_{\mathrm{k}}\right)^{\mathrm{T}} \widetilde{\mathrm{G}_{\mathrm{k}}}\left(\widetilde{\mathrm{G}}^{\frac{1}{2}} \mathrm{~s}_{\mathrm{k}}\right)}{\left(\mathrm{s}_{\mathrm{k}}{\widetilde{\mathrm{G}_{\mathrm{k}}}}^{\frac{1}{2}}\right)^{\mathrm{T}}\left({\widetilde{\mathrm{G}_{\mathrm{k}}}}^{\frac{1}{2}} \mathrm{~s}_{\mathrm{k}}\right)}$

$$
\begin{aligned}
& \mathrm{s}_{\mathrm{k}}^{\mathrm{T}\left(\frac{\left(\mathrm{B}_{\mathrm{k}} \mathrm{s}_{\mathrm{k}}+\mathrm{s}_{\mathrm{k}}\right) \mathrm{y}_{\mathrm{k}}^{\mathrm{T}}}{\mathrm{y}_{\mathrm{k}}^{\mathrm{T}} \mathrm{y}_{\mathrm{k}}}\right) \mathrm{y}_{\mathrm{k}}} \\
& =\frac{\mathrm{s}_{\mathrm{k}}^{\mathrm{T}} \widetilde{\mathrm{G}}_{\mathrm{k}}{ }^{\frac{1}{2}}{\widetilde{\mathrm{G}_{\mathrm{k}}}}^{\frac{1}{2}} \widetilde{\mathrm{G}}_{\mathrm{k}}{ }^{\frac{1}{2}} \widetilde{\mathrm{G}}_{\mathrm{k}}{ }^{\frac{1}{2}} \mathrm{~s}_{\mathrm{k}}}{\mathrm{s}_{\mathrm{k}}^{\mathrm{T}}{\widetilde{\mathrm{G}_{\mathrm{k}}}}^{\frac{1}{2}}{\widetilde{\mathrm{G}_{\mathrm{k}}}}^{\frac{1}{2}} \mathrm{~s}_{\mathrm{k}}}
\end{aligned}
$$

By substituting equation (15) in equation (16), we get

$$
\frac{\left\|\left(\frac{\left(B_{k} s_{k}+s_{k}\right) y_{k}^{T}}{y_{k}^{T} y_{k}}\right) y_{k}\right\| \|^{2}}{s_{k}^{T}\left(\frac{\left(B_{k} s_{k}+s_{k}\right) y_{k}^{T}}{y_{k}^{T} y_{k}}\right) y_{k}}=\frac{v_{k}^{T} \widetilde{G_{k}} v_{k}}{v_{k}^{T} v_{k}}
$$

and from (12) $\mathrm{u}^{\mathrm{T}} \nabla^{2} \mathrm{f}\left(\mathrm{x}^{*}\right) \mathrm{u} \leq \mathrm{C}\|\mathrm{u}\|^{2}$, we know $\|\mathrm{u}\|^{2}>0$. Then by dividing both sides of (12), we get:

$\frac{v_{k}^{T} \widetilde{G_{k}} v_{k}}{\left\|u_{k}\right\|^{2}} \leq C$ that mean $\frac{v_{k}^{T} \widetilde{G_{k}} v_{k}}{v_{k}^{T} v_{k}} \leq C$

Then from equation (17), we get : 
$\frac{\left\|\left(\frac{\left(B_{k} s_{k}+s_{k}\right) y_{k}^{T}}{y_{k}^{T} y_{k}}\right) y_{k}\right\|^{2}}{s_{k}^{T}\left(\frac{\left(B_{k} s_{k}+s_{k}\right) y_{k}^{T}}{y_{k}^{T} y_{k}}\right) y_{k}} \leq C$

From equation (13), we get :

$$
\begin{aligned}
&\left\|\left(\frac{\left(B_{k} s_{k}+s_{k}\right) y_{k}^{T}}{y_{k}^{T} y_{k}}\right) y_{k}\right\|=\left\|\widetilde{\mathrm{G}_{k}} s_{k}\right\| \\
&\left\|\left(\frac{\left(B_{k} s_{k}+s_{k}\right) y_{k}^{T}}{y_{k}^{T} y_{k}}\right) y_{k}\right\| \leq\left\|\widetilde{G_{k}}\right\|\left\|s_{k}\right\|, \quad\left\|s_{k}\right\| \\
& \leq\left\|\widetilde{G_{k}}-1\right\|\left\|\left(\frac{\left(B_{k} s_{k}+s_{k}\right) y_{k}^{T}}{y_{k}^{T} y_{k}}\right) y_{k}\right\|
\end{aligned}
$$

Which gives :

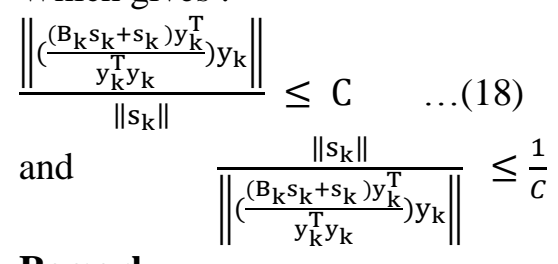

\section{Remark}

We use $F(x)$ and $F^{\prime}(x)$ to mean $\nabla f(x)$ and $\nabla^{2} \mathrm{f}(\mathrm{x})$ respectively.

\section{Assumption 2, [6]}

(A): $\mathrm{F}: \mathrm{R}^{\mathrm{n}} \rightarrow \mathrm{R}^{\mathrm{n}}$ is continuosly differentiable in open convex set $\mathrm{D} \subset \mathrm{R}^{\mathrm{n}}$.

(B): There is $\mathrm{x}^{*}$ in $\mathrm{D}$, such that $\mathrm{F}\left(\mathrm{x}^{*}\right)=0$ and $\mathrm{F}^{\prime}\left(\mathrm{x}^{*}\right)$ is non singular.

(C): $\mathrm{F}^{\prime}(\mathrm{x})$ satisfies the Lipschitz condition at $\mathrm{x}^{*}$, that is, there exists a constant

$$
\beta>0 \text { such that }
$$

$$
\left\|F^{\prime}(x)-F^{\prime}\left(x^{*}\right)\right\| \leq \beta\left\|x-x^{*}\right\|, \quad x^{*}, x \in D
$$

We begin with some general converged results.

Lemma (1), [6]

Let $\mathrm{f}: \mathrm{R}^{\mathrm{n}} \rightarrow \mathrm{R}$ satisfy Assumption (1), then

$$
\frac{\left\|s_{k}\right\|}{\left\|y_{k}\right\|}, \frac{\left\|y_{k}\right\|}{\left\|s_{k}\right\|}, \frac{s_{k}^{T} y_{k}}{\left\|s_{k}\right\|^{2}}, \frac{s_{k}^{T} y_{k}}{\left\|y_{k}\right\|^{2}}, \frac{\left\|y_{k}\right\|^{2}}{s_{k}^{T} y_{k}}, \text { are }
$$

bounded."

\section{Lemma (2)}

Let $\quad f: R^{n} \rightarrow R \quad$ satisfy Assumption (1) then,

$\frac{\left\|s_{k}\right\|}{\left\|y^{*}\right\|}, \frac{\left\|y^{*}\right\|}{\left\|s_{k}\right\|}, \frac{s_{k}^{T} y^{*}}{\left\|s_{k}\right\|^{2}}, \frac{s_{k}^{T} y^{*}}{\left\|y^{*}\right\|^{2}}, \frac{\left\|y^{*}\right\|^{2}}{s_{k}^{T} y^{*}}, \frac{s_{k}^{T} B_{k} s_{k}}{\left\|s_{k}\right\|^{2}}$,

$\frac{s_{k}^{T} B_{k} H_{k} B_{k} s_{k}}{\left\|s_{k}\right\|^{2}}, \frac{s_{k}^{T} B_{k} H_{k} y^{*}}{\left\|s_{k}\right\|^{2}}, \frac{s_{k}^{T} H_{k} s_{k}}{\left\|s_{k}\right\|^{2}}$ are bounded.

\section{Lemma (3), [6]}

Under exact line search, $\sum\left\|s_{\mathrm{k}}\right\|^{2}$ and $\sum\left\|\mathrm{y}_{\mathrm{k}}\right\|^{2}$ are convergent.

\section{Lemma (4)}

Under exact or inexact line search, $\sum\left\|\mathrm{s}_{\mathrm{k}}\right\|^{2}$ and $\sum\left\|\mathrm{y}^{*}\right\|^{2}$ are convergent.

Proof: $\sum\left\|s_{k}\right\|^{2}$ is convergent. where $f\left(x^{*}\right)$ is the minimum of $f(x)$.

Now, we will prove $\sum\left\|\left(\frac{\left(B_{k} s_{k}+s_{k}\right) y_{k}^{T}}{y_{k}^{T} y_{k}}\right) y_{k}\right\|^{2}$ is convergent, from lemma (1), we have
$\frac{\left\|\left(\frac{\left(B_{k} s_{k}+s_{k}\right) y_{k}^{T}}{y_{k}^{T} y_{k}}\right) y_{k}\right\|^{2}}{\left\|s_{k}\right\|^{2}}$ is bounded, then $\exists C$ is a positive number which is independent of $\mathrm{k}$ such that it satisfy (18). And from (18), we obtain $\|\left(\left(\frac{\left(B_{k} s_{k}+s_{k}\right) y_{k}^{T}}{y_{k}^{T} y_{k}}\right) y_{k}\left\|^{2} \leq C^{2}\right\| s_{k} \|^{2}\right.$

Since $\sum\left\|s_{\mathrm{k}}\right\|^{2}$ is convergent, we get $\left\|\left(\frac{\left(B_{k} s_{k}+s_{k}\right) y_{k}^{T}}{y_{k}^{T} y_{k}}\right) y_{k}\right\|^{2}$ is bounded, then

$\sum_{\mathrm{k}=0}^{\infty}\left\|\left(\frac{\left(\mathrm{B}_{\mathrm{k}} \mathrm{s}_{\mathrm{k}}+\mathrm{s}_{\mathrm{k}}\right) \mathrm{y}_{\mathrm{k}}^{\mathrm{T}}}{\mathrm{y}_{\mathrm{k}}^{\mathrm{T}} \mathrm{y}_{\mathrm{k}}}\right) \mathrm{y}_{\mathrm{k}}\right\|^{2} \leq \mathrm{C}^{2} \sum_{\mathrm{k}=0}^{\infty}\left\|\mathrm{s}_{\mathrm{k}}\right\|^{2}$

Which implies $\sum\left\|\left(\frac{\left(B_{k} s_{k}+s_{k}\right) y_{k}^{T}}{y_{k}^{T} y_{k}}\right) y_{k}\right\|^{2} \quad$ is convergent, where $f\left(x^{*}\right)$ is the minmum value of $f(x)$.

\section{Lemma (5)}

For SPBR1 update, the determinant of the next Hessian matrix is given by

$\left|B_{k+1}\right|=\left|B_{k}\right|\left[\frac{s_{k} w_{k}}{s_{k}^{T} s_{k}}\right]$

where

$\mathrm{s}_{\mathrm{k}}=\left(\left(\frac{\left(\mathrm{B}_{\mathrm{k}} \mathrm{s}_{\mathrm{k}}+\mathrm{s}_{\mathrm{k}}\right) \mathrm{y}_{\mathrm{k}}^{\mathrm{T}}}{\mathrm{y}_{\mathrm{k}}^{\mathrm{T}} \mathrm{y}_{\mathrm{k}}}\right) \mathrm{y}_{\mathrm{k}}-\mathrm{B}_{\mathrm{k}} \mathrm{s}_{\mathrm{k}}\right)$,

$\mathrm{w}_{\mathrm{k}}=\mathrm{s}_{\mathrm{k}}-\mathrm{H}_{\mathrm{k}} \mathrm{s}_{\mathrm{k}}$, and $H_{k}=B_{k}^{-1}$.

Proof: we can prove from the SPBR1 update (10)

$\left|\mathrm{B}_{\mathrm{k}+1}\right|$

$=\mid \mathrm{B}_{\mathrm{k}}$

$+\frac{\left(\left(\frac{\left(B_{k} s_{k}+s_{k}\right) y_{k}^{T}}{y_{k}^{T} y_{k}}\right) y_{k}-B_{k} s_{k}\right) s_{k}^{T}}{s_{k}^{T} s_{k}} \mid$

$\left|B_{k+1}\right|=\left|B_{k}-\frac{s_{k} s_{k}^{T}}{s_{k}^{T} s_{k}}\right|$

$\left|\mathrm{B}_{\mathrm{k}+1}\right|=\left|\mathrm{B}_{\mathrm{k}}\right|\left|\mathrm{I}-\frac{\mathrm{L}_{\mathrm{k}}^{-1} \mathrm{~s}_{\mathrm{k}} \mathrm{s}_{\mathrm{k}}^{\mathrm{T}} \mathrm{L}_{\mathrm{k}}^{\mathrm{T}^{-1}}}{\mathrm{~s}_{\mathrm{k}}^{\mathrm{T}} \mathrm{s}_{\mathrm{k}}}\right|$

Where $\mathrm{B}_{\mathrm{k}}=\mathrm{L}_{\mathrm{k}} \mathrm{L}_{\mathrm{k}}^{\mathrm{T}}$

$\left|B_{k+1}\right|=\left|B_{k}\right|\left|I-\frac{\left(L_{k}^{-1} s_{k}\right)\left(L_{k}^{-1} s_{k}\right)^{T}}{s_{k}^{T} s_{k}}\right|$

Let $\quad u_{k}=\frac{L_{k}^{-1} s_{k}}{s_{k}^{T} s_{k}}, \quad v_{k}=L_{k}^{-1} s_{k}, \quad \ldots$ (20)

By formula $\left(\left|\mathrm{I}+\mathrm{u}_{\mathrm{k}} \mathrm{v}_{\mathrm{k}}^{\mathrm{T}}\right|=1+\mathrm{u}_{\mathrm{k}}^{\mathrm{T}} \mathrm{v}_{\mathrm{k}}\right) \quad$ and equation (20)

$\left|\mathrm{B}_{\mathrm{k}+1}\right|=\left|\mathrm{B}_{\mathrm{k}}\right|\left[1-\frac{\left(\mathrm{L}_{\mathrm{k}}^{-1} \mathrm{~s}_{\mathrm{k}}\right)^{\mathrm{T}}\left(\mathrm{L}_{\mathrm{k}}^{-1} \mathrm{~s}_{\mathrm{k}}\right)}{\mathrm{s}_{\mathrm{k}}^{\mathrm{T}} \mathrm{s}_{\mathrm{k}}}\right] \quad \ldots$

$\left|\mathrm{B}_{\mathrm{k}+1}\right|=\left|\mathrm{B}_{\mathrm{k}}\right|\left[1-\frac{\left(\mathrm{L}_{\mathrm{k}}^{-1} \mathrm{~s}_{\mathrm{k}}\right)^{\mathrm{T}}\left(\mathrm{L}_{\mathrm{k}}^{-1} \mathrm{~s}_{\mathrm{k}}\right)}{\mathrm{s}_{\mathrm{k}}^{\mathrm{T}} \mathrm{s}_{\mathrm{k}}}\right]$

$\left|\mathrm{B}_{\mathrm{k}+1}\right|=\left|\mathrm{B}_{\mathrm{k}}\right|\left[1-\frac{\mathrm{s}_{\mathrm{k}}^{\mathrm{T}} \mathrm{H}_{\mathrm{k}} \mathrm{s}_{\mathrm{k}}}{\mathrm{s}_{\mathrm{k}}^{\mathrm{T}} \mathrm{s}_{\mathrm{k}}}\right]$

Where $\mathrm{H}_{\mathrm{k}}=\mathrm{L}_{\mathrm{k}}^{-1^{\mathrm{T}}} \mathrm{L}_{\mathrm{k}}^{-1}$

$\left|B_{k+1}\right|=\left|B_{k}\right|\left[\frac{s_{k}^{T} s_{k}-s_{k}^{T} H_{k} s_{k}}{s_{k}^{T} s_{k}}\right]$ 


$$
\left|B_{k+1}\right|=\left|B_{k}\right|\left[\frac{s_{k}^{T}\left(s_{k}-H_{k} s_{k}\right)}{s_{k}^{T} s_{k}}\right]
$$

Let $\mathrm{w}_{\mathrm{k}}=\mathrm{s}_{\mathrm{k}}-\mathrm{H}_{\mathrm{k}} \mathrm{s}_{\mathrm{k}}$, we get:

$$
\left|\mathrm{B}_{\mathrm{k}+1}\right|=\left|\mathrm{B}_{\mathrm{k}}\right|\left[\frac{s_{\mathrm{k}}^{\mathrm{T}} \mathrm{w}_{\mathrm{k}}}{\mathrm{s}_{\mathrm{k}}^{\mathrm{T}} \mathrm{s}_{\mathrm{k}}}\right]
$$

\section{Theorem (4)}

Suppose that $\mathrm{f}(\mathrm{x})$ satisfies Assumption (1). Then under exact or inexact line search the sequence $\left\{x_{\mathrm{k}}\right\}$ generated by SPBR1 update converges to the minimizer $\mathrm{x}^{*}$ of $\mathrm{f}$.

Proof :

Consider SPBR1 update (10) of Hessian matrix

$B_{k+1}=B_{k}+\frac{\left(\left(\frac{\left(B_{k} s_{k}+s_{k}\right) y_{k}^{T}}{y_{k}^{T} y_{k}}\right) y_{k}-B_{k} s_{k}\right) s_{k}{ }^{T}}{s_{k}{ }^{T} s_{k}}$

Subtracting equation (6), in (10), we get :

$\mathrm{B}_{\mathrm{k}+1}=\mathrm{B}_{\mathrm{k}}+\frac{\mathrm{s}_{\mathrm{k}} \mathrm{s}_{\mathrm{k}}^{\mathrm{T}}}{\mathrm{s}_{\mathrm{k}}^{\mathrm{T}} \mathrm{s}_{\mathrm{k}}}$

By taking the trace operator for both sides of equation (23), we get:"

$\operatorname{tr}\left(B_{k+1}\right)=\operatorname{tr}\left(B_{k}\right)+\frac{s_{k}^{T} s_{k}}{s_{k}^{T} s_{k}}$

By taking the In operator for equation (22), we get:

$\ln \left(\left|B_{k+1}\right|\right)=\ln \left(\left|B_{k}\right| \frac{s_{k}^{T} w_{k}}{s_{k}^{T} s_{k}}\right)$

Define

$$
\psi\left(B_{k}\right)=\operatorname{tr}\left(B_{k}\right)-\ln \left(\left|B_{k}\right|\right) \geq 0
$$

(26)

By replacing $B_{k}$ by $B_{k+1}$ in equation

$$
\psi\left(B_{k+1}\right)=\operatorname{tr}\left(B_{k+1}\right)-\ln \left(\left|B_{k+1}\right|\right)
$$

By substitution (25), and (26), in (27), we get:

$$
\begin{aligned}
\Psi\left(B_{\mathrm{k}+1}\right) & =\operatorname{tr}\left(B_{k}\right)+1-\ln \left(\left|B_{k}\right| \frac{s_{k}^{T} w_{k}}{s_{k}^{T} s_{k}}\right) \\
= & \operatorname{tr}\left(B_{k}\right)+1-\ln \left(\left|B_{k}\right| \frac{s_{k}^{T}\left(s_{k}-H_{k} s_{k}\right)}{s_{k}^{T} s_{k}}\right) \\
= & \operatorname{tr}\left(B_{k}\right)+1-\ln \left(\left|B_{k}\right|\right)- \\
\ln \left(s_{k}^{T} s_{k}\right. & \left.-s_{k}^{T} H_{k} s_{k}\right)+\ln \left(s_{k}^{T} s_{k}\right)
\end{aligned}
$$

Substituting (26) in (28), we get:

$\psi\left(B_{k+1}\right)=\psi\left(B_{k}\right)+1-\ln \left(s_{k}^{T} s_{k}-s_{k}^{T} H_{k} s_{k}\right)+$ $\ln \left(\mathrm{s}_{\mathrm{k}}^{\mathrm{T}} \mathrm{s}_{\mathrm{k}}\right)$

Without loss the generality of the prove we can assume that $\ln \left(\mathrm{s}_{\mathrm{k}}^{\mathrm{T}} \mathrm{s}_{\mathrm{k}}-\mathrm{s}_{\mathrm{k}}^{\mathrm{T}} \mathrm{H}_{\mathrm{k}} \mathrm{s}_{\mathrm{k}}\right)$ is positive. $\psi\left(\mathrm{B}_{\mathrm{k}+1}\right) \leq \psi\left(\mathrm{B}_{\mathrm{k}}\right)+1+\ln \left(\mathrm{s}_{\mathrm{k}}^{\mathrm{T}} \mathrm{s}_{\mathrm{k}}\right) \quad \ldots$

Define $\cos \vartheta_{\mathrm{k}}=\frac{s_{\mathrm{k}}^{\mathrm{T}} \mathrm{B}_{\mathrm{k}} \mathrm{s}_{\mathrm{k}}}{\left\|\mathrm{s}_{\mathrm{k}}\right\|\left\|\mathrm{B}_{\mathrm{k}} \mathrm{s}_{\mathrm{k}}\right\|}$ and $\mathrm{q}_{\mathrm{k}}=\frac{\left(\mathrm{s}_{\mathrm{k}}^{\mathrm{T}} \mathrm{B}_{\mathrm{k}} \mathrm{s}_{\mathrm{k}}\right)^{2}}{\left\|\mathrm{~s}_{\mathrm{k}}\right\|^{4}\left\|\mathrm{~B}_{\mathrm{k}} \mathrm{s}_{\mathrm{k}}\right\|^{2}}$ $\mathrm{s}_{\mathrm{k}}^{\mathrm{T}} \mathrm{s}_{\mathrm{k}}=\frac{\cos ^{2} \vartheta_{\mathrm{k}}}{\mathrm{q}_{\mathrm{k}}}$

Substituting (31), in (30) we get:

$\psi\left(B_{k+1}\right)=\psi\left(B_{k}\right)+1+\ln \left(\frac{\cos ^{2} \vartheta_{k}}{q_{k}}\right)$

$\psi\left(B_{k+1}\right) \leq \psi\left(B_{k}\right)+1+\ln \left(\cos ^{2} \vartheta_{k}\right)-\ln \left(q_{k}\right)$ ...(32)

Summing from $\mathrm{j}=1$ up to $\mathrm{k}$

$$
\begin{aligned}
\sum_{\mathrm{j}=1}^{\mathrm{k}} \psi\left(\mathrm{B}_{\mathrm{j}+1}\right) \leq \sum_{\mathrm{j}=1}^{\mathrm{k}} & \psi\left(\mathrm{B}_{\mathrm{j}}\right) \\
& +\sum_{\mathrm{j}=1}^{\mathrm{k}}\left(1-\ln \left(\mathrm{q}_{\mathrm{k}}\right)\right)+\sum_{\mathrm{j}=1}^{\mathrm{k}} \ln \left(\cos ^{2} \vartheta_{\mathrm{j}}\right) \\
0<\psi\left(\mathrm{B}_{2}\right)+\cdots & +\left(\mathrm{B}_{\mathrm{k}+1}\right) \\
\leq & \psi\left(\mathrm{B}_{1}\right)+\cdots+\psi\left(\mathrm{B}_{\mathrm{k}}\right)+\mathrm{C} . \mathrm{k} \\
& +\sum_{\mathrm{j}=1}^{\mathrm{k}} \ln \left(\cos ^{2} \vartheta_{\mathrm{j}}\right)
\end{aligned}
$$$$
0<\psi\left(B_{k+1}\right) \leq \psi\left(B_{1}\right)+C . k+\sum_{j=1}^{k} \ln \left(\cos ^{2} \vartheta_{j}\right)
$$

Where the constant $C=1-\ln \left(q_{k}\right)$ is assumed to be positive without loss of generality and from Zoutendijk condition [4], we have:

$$
\lim _{\mathrm{k} \rightarrow \infty} \mathrm{F}\left(\mathrm{x}_{\mathrm{k}}\right) \cos \vartheta_{\mathrm{k}}=0
$$

If $\vartheta_{\mathrm{k}}$ is bounded away from $90^{\circ}, \exists \mu \in \mathrm{R}^{+} \exists$ $\cos \vartheta_{k}>\mu>0$, for sufficient large $k$ and hence $\mathrm{F}\left(\mathrm{x}_{\mathrm{k}}\right) \rightarrow 0$ and the proof is complete.

Now, assume by contradiction that $\cos \vartheta_{\mathrm{k}} \rightarrow 0$, then $\exists \mathrm{k}_{1}>0 \exists \forall \mathrm{j}>\mathrm{k}_{1}$ we have

$\ln \left(\cos ^{2} \vartheta_{\mathrm{j}}\right)<-2 \mathrm{C}$

By (33), we get:

$0<\psi\left(B_{1}\right)+C . k+\sum_{j=1}^{\mathrm{k}_{1}} \ln \left(\cos ^{2} \vartheta_{\mathrm{j}}\right)+$

$\sum_{\mathrm{j}=\mathrm{k}_{1}+1}^{\mathrm{k}} \ln \left(\cos ^{2} \vartheta_{\mathrm{j}}\right) \quad, \mathrm{k}_{1}+1<\mathrm{k}$

By use the equation (34), in (35), we have :

$0<\psi\left(B_{1}\right)+\mathrm{C} . \mathrm{k}+\sum_{\mathrm{j}=1}^{\mathrm{k}_{1}} \ln \left(\cos ^{2} \vartheta_{\mathrm{j}}\right)+$

$\sum_{\mathrm{j}=\mathrm{k}_{1}+1}^{\mathrm{k}}(-2 \mathrm{C})$

$0<\psi\left(B_{1}\right)+\sum_{j=1}^{k_{1}} \ln \left(\cos ^{2} \vartheta_{j}\right)+2 C . k_{1}-C k$.

Hence $\psi\left(B_{k+1}\right)<0$ for sufficiently large $\mathrm{k}$ which is a contradiction then, $\cos ^{2} \vartheta_{j} \rightarrow 0$ is not true. Then $\lim _{\mathrm{k} \rightarrow \infty} \inf \mathrm{F}\left(\mathrm{x}_{\mathrm{k}}\right) \rightarrow 0$ or $\mathrm{x}_{\mathrm{k}} \rightarrow \mathrm{x}^{*}$.

\section{Numerical Results:}

This section is devoted to numerical experiments. Our purpose is to check whether the SPBR1 algorithm provides improvements on the corresponding standard Broyden algorithm. The programs are written in MATLAB with single precision. The test functions commonly use unconstrained test problems with the same starting point and a summary of which is given in Table (1). The test functions are chosen as follows:

1 - Least square equation for two dimention [2]

$\mathrm{f}(\mathrm{x})=\left(1-\mathrm{x}_{1}\right)^{2}+\left(1-\mathrm{x}_{2}\right)^{2}$.

$2-$ A quartic function . [2]

$f(x)=\sum_{i=1}^{4}\left(10^{i-1} x_{i}^{4}+x_{i}^{3}+10^{1-i} x_{i}^{2}\right)$.

3 - Rosenbroc'k cliff function [3]

$\mathrm{f}(\mathrm{x})=10^{-4}\left(\mathrm{x}_{1}-3\right)^{2}-\left(\mathrm{x}_{1}-\mathrm{x}_{2}\right)+\mathrm{e}^{20\left(\mathrm{x}_{1}-\mathrm{x}_{2}\right)}$.

$4-$ Generalized Edger function [2]

$\mathrm{f}(\mathrm{x})=\sum_{\mathrm{i}=1}^{\mathrm{n} / 2}\left[\left(\mathrm{x}_{2 \mathrm{i}-1}-2\right)^{4}+\left(\mathrm{x}_{2 \mathrm{i}-1}-2\right)^{2} \mathrm{x}_{2 \mathrm{i}}^{2}+\right.$

$\left.\left(x_{2 i}+1\right)^{2}\right]$. 
5 - Rosen rock's function [3]

$f(x)=\sum_{i=1}^{n / 2}\left[100\left(x_{i}-x_{i}^{3}\right)^{2}+\left(1-x_{i}\right)^{2}\right]$.

6 - Trigonometric function [3]

$\mathrm{f}(\mathrm{x})=\sum_{\mathrm{i}=1}^{\mathrm{n}}\left[\mathrm{n}-\sum_{\mathrm{j}=1}^{\mathrm{n}} \cos \mathrm{x}_{\mathrm{j}}+\mathrm{i}\left(1-\cos \mathrm{x}_{\mathrm{i}}\right)-\right.$

$\left.\sin x_{i}+e^{x i}-1\right]^{2}$.

7 - Watson function [2]

$\mathrm{F}(\mathrm{x})=\sum_{\mathrm{i}=1}^{\mathrm{j}} \mathrm{f}_{\mathrm{i}}^{2}(\mathrm{x})$

$\mathrm{f}_{\mathrm{i}}(\mathrm{x})=\sum_{\mathrm{j}=2}^{3}(\mathrm{j}-1) \mathrm{x}_{\mathrm{j}} \mathrm{t}_{\mathrm{j}}^{\mathrm{j}-2}-\left(\sum_{\mathrm{j}=1}^{3} \mathrm{x}_{\mathrm{j}} \mathrm{t}_{\mathrm{j}}^{\mathrm{j}-1}\right)^{2}-1$.

and $t_{j}=\frac{i}{29}$.

8-Trigonometric function [2]

$f(x)=100\left(x_{2}-\sin x_{1}\right)^{2}+0.25 x_{1}^{2}$
9- Rosen function [1]

$f(x)=100\left(x_{2}-x_{1}^{2}\right)^{2}+\left(1-x_{1}\right)^{2}$

10- Cubic function [1]

$f(x)=100\left(x_{2}-x_{1}^{3}\right)^{2}+\left(1-x_{1}\right)^{2}$

11- Trigonometric function [6]

$f(x)=\sum_{i=1}^{n}\left[n+i\left(1-\cos x_{i}\right)-\sin x_{i}-\sum_{j=1}^{n} \cos x_{j}\right]^{2}$

12-Extended Rosenbrock function [6]

$f(x)=\sum_{i=1}^{n-1}\left[100\left(x_{i+1}-x_{i}^{2}\right)^{2}+\left(1-x_{i}\right)^{2}\right]$

13 - Least sequare function [6]

$f(x)=\left(x_{1}-2\right)^{2}+\left(x_{2}-1\right)^{2}$

Table 1.Numerical results for Broyden and SPBR1 update

\begin{tabular}{|c|c|c|c|c|c|c|}
\hline \multirow{2}{*}{ Fun. } & \multirow{2}{*}{ Starting point } & \multirow{2}{*}{ Dim. } & \multicolumn{2}{|c|}{ Broyden } & \multicolumn{2}{|c|}{ SPBR1 } \\
\hline & & & Feval & Iter. & Feval & Iter. \\
\hline 1 & $(-15 ; 5)^{\mathrm{T}}$ & 2 & $2.8692 \mathrm{e}-015$ & 2 & $2.8692 \mathrm{e}-015$ & 2 \\
\hline 1 & $(0.5 ;-0.8)^{\mathrm{T}}$ & 2 & $5.0919 \mathrm{e}-017$ & 2 & $5.0919 \mathrm{e}-017$ & 2 \\
\hline 1 & $(1 / 5 ; 1 / 5)^{\mathrm{T}}$ & 2 & $3.7024 \mathrm{e}-017$ & 2 & $3.7024 \mathrm{e}-017$ & 2 \\
\hline 2 & $(-1 ; 0 ; 0 ; 0)^{\mathrm{T}}$ & 4 & -0.1055 & 3 & -0.1055 & 3 \\
\hline 2 & $(-2 ; 0 ; 0 ; 0)^{\mathrm{T}}$ & 4 & -0.1055 & 3 & -0.1055 & 3 \\
\hline 2 & $(0 ; 5 ; 0 ; 5)^{\mathrm{T}}$ & 4 & 11.6337 & 67 & -2.3963 & 12 \\
\hline 3 & $(12 ; 4.8)^{\mathrm{T}}$ & 2 & Nan & 2 & $1.9485 \mathrm{e}+016$ & 2 \\
\hline 3 & $(12 ; 5)^{\mathrm{T}}$ & 2 & Nan & 2 & $1.0458 \mathrm{e}+059$ & 2 \\
\hline 3 & $(12 ; 4.9)^{\mathrm{T}}$ & 2 & Nan & 2 & $5.7100 \mathrm{e}+060$ & 2 \\
\hline 4 & $(2 ;-2)^{\mathrm{T}}$ & 2 & $1.5103 \mathrm{e}-0.16$ & 2 & $1.5103 \mathrm{e}-016$ & 2 \\
\hline 4 & $(1 / 4 ; 1 / 4)^{\mathrm{T}}$ & 2 & $4.4951 \mathrm{e}-008$ & 9 & $2.4152 \mathrm{e}-010$ & 6 \\
\hline 4 & $(0 ; 1)^{\mathrm{T}}$ & 2 & $5.7967 \mathrm{e}-010$ & 13 & $5.004 \mathrm{e}-010$ & 7 \\
\hline 5 & $(5 ; 2)^{\mathrm{T}}$ & 2 & 0.9901 & 7 & 0.9901 & 5 \\
\hline 5 & $(1.2 ; 1.2)^{\mathrm{T}}$ & 2 & $5.2958 \mathrm{e}-015$ & 3 & $1.8357 \mathrm{e}-015$ & 3 \\
\hline 5 & $(1.2 ; 0)^{\mathrm{T}}$ & 2 & $5.2958 \mathrm{e}-015$ & 3 & $5.2958 \mathrm{e}-015$ & 3 \\
\hline 6 & $(0.5 ; 0.5)^{\mathrm{T}}$ & 2 & $3.9206 \mathrm{e}-005$ & 31 & $1.8340 \mathrm{e}-006$ & 4 \\
\hline 6 & $(2 ;-2)^{\mathrm{T}}$ & 2 & 0.0091 & 39 & $6.7395 \mathrm{e}-007$ & 5 \\
\hline 6 & $(-5 ; 5)^{\mathrm{T}}$ & 2 & 0.0153 & 34 & $1.4279 \mathrm{e}-006$ & 18 \\
\hline 7 & $\left(\frac{1}{8} ; \frac{1}{8} ; \frac{1}{8}\right)^{\mathrm{T}}$ & 3 & $6.9016 \mathrm{e}-017$ & 3 & $2.8104 \mathrm{e}-018$ & 3 \\
\hline 7 & $(-1 ; 0 ; 0)^{\mathrm{T}}$ & 3 & $1.6335 \mathrm{e}-016$ & 4 & $3.2750 \mathrm{e}-019$ & 4 \\
\hline 7 & $(0.5 ; 0.5 ; 0.5)^{\mathrm{T}}$ & 3 & $4.0131 \mathrm{e}-013$ & 3 & $2.6114 \mathrm{e}-018$ & 3 \\
\hline 8 & $\left(\frac{2}{3} ; \frac{1}{3}\right)^{\mathrm{T}}$ & 2 & $2.4564 \mathrm{e}-005$ & 19 & $1.7914 \mathrm{e}-005$ & 10 \\
\hline 8 & $\left(\frac{1}{2} ; \frac{1}{4}\right)^{\mathrm{T}}$ & 2 & Nan & 62 & $4.8289 \mathrm{e}-005$ & 32 \\
\hline 8 & $(3.3 ; 6.6)^{\mathrm{T}}$ & 2 & $2.0472 \mathrm{e}-004$ & 5 & $7.9348 \mathrm{e}-008$ & 10 \\
\hline 9 & $(1.1 ; 1.1)^{\mathrm{T}}$ & 2 & 0.0011 & 32 & $4.7181 \mathrm{e}-007$ & 6 \\
\hline 9 & $(1 ; 1)^{\mathrm{T}}$ & 2 & 0 & 1 & 0 & 1 \\
\hline 9 & $(1.2 ; 1)^{\mathrm{T}}$ & 2 & $3.2764 \mathrm{e}-006$ & 21 & $3.2764 \mathrm{e}-006$ & 21 \\
\hline 10 & $(1.5 ; 1)^{\mathrm{T}}$ & 2 & 0.0496 & 37 & $7.4441 \mathrm{e}-006$ & 9 \\
\hline 10 & $(1 ; 1.2)^{\mathrm{T}}$ & 2 & 0.0026 & 8 & $1.2313 \mathrm{e}-005$ & 26 \\
\hline 10 & $(-15 ; 5)^{\mathrm{T}}$ & 2 & Nan & 44 & $2.9365 \mathrm{e}-006$ & 11 \\
\hline 11 & $(0.5 ; 0.5)^{\mathrm{T}}$ & 2 & 0.0043 & 30 & $1.1238 \mathrm{e}-006$ & 10 \\
\hline 11 & $(-1 ; 0)^{\mathrm{T}}$ & 2 & $7.0570 \mathrm{e}-008$ & 7 & $2.0684 \mathrm{e}-012$ & 4 \\
\hline 11 & $\left(\frac{1}{4} ; \frac{1}{4}\right)^{\mathrm{T}}$ & 2 & $4.0107 \mathrm{e}-008$ & 13 & $1.70420 \mathrm{e}-009$ & 5 \\
\hline 12 & $(0 ; 11)^{\mathrm{T}}$ & 2 & $9.8078 \mathrm{e}-006$ & 19 & 0.3805 & 58 \\
\hline 12 & $(1.1 ; 1.1)^{\mathrm{T}}$ & 2 & 0.0011 & 32 & $4.7181 \mathrm{e}-007$ & 6 \\
\hline 12 & $(1 ; 1 ; 1 ; 1 ; 1 ; 1)$ & 6 & 0 & 1 & 0 & 1 \\
\hline 13 & $\left(\frac{1}{4} ; \frac{1}{4}\right)^{\mathrm{T}}$ & 2 & $2.0118 \mathrm{e}-018$ & 2 & $2.0118 \mathrm{e}-018$ & 2 \\
\hline 13 & $(10 ; 3)^{\mathrm{T}}$ & 2 & $2.3306 \mathrm{e}-015$ & 2 & $2.3306 \mathrm{e}-015$ & 2 \\
\hline 13 & $(4 ; 5)^{\mathrm{T}}$ & 2 & $5.4879 \mathrm{e}-018$ & 2 & $5.4879 \mathrm{e}-018$ & 2 \\
\hline
\end{tabular}


Remark: the mean of Nan is $\frac{\infty}{\infty}$ or $0 . \infty$ or $\frac{0}{0}$.

From Table (1), it's clear that SPBR1 has a performance better than Broyden update, and we can note that the Broyden update cannot solve problem 3 at all starting points because the singularity of the Hessian matrix but SPBR1 can get the minimum of this problem at all starting points. Moreover, the Broyden update in a several test problems cannot get the minimizer and the program break the loop before getting the minimizer but SPBR1 can continue to the minimizer and that's clear in problems 2, 4, 6,8, and 10.

\section{Conclusion:}

In this paper, the Broyden update is modified to guarantee the symmetric and positively definite properties and the so called symmetric positive definite Broyden update. The convergence of the proposed method is established and the numerical results are reported in table (1). It can be seen that the proposed method in most tests is better than the Broyden method.

\section{Conflicts of Interest: None.}

\section{References:}

1. Al Bayati Abass Y., Muhamed Sabah A. A New SelfScaling Variable Metric Algorithm for Nonlinear Optimization. IJMA. 2013; 4(5) : 184-191.

2. Mahmood Saad S., Farqad H. On Extended Symmetric Rank One Update for Unconstrained Optimization. Journal of College of Education . 2017; 6(1): 206-220 .

3. Mahmood Saad S., Shnywer Samira H. On Modified DFP Update for Unconstrained Optimization. AJAM . 2017; 5(1): 19-30.

4. Nocedal J. Wright, Stephen J. Numerical Optimization. $2^{\text {nd }}$ edition, Springer Science + Business Media, LLC. 2006.

5. Osingua I. A., Yusuff S. O. Construction of a Broyden -Like Method for Nonlinear Systems of Equation. Annals. Computer Science Series. 2017; 15(2): 128-135.

6. Wenyu Sun, Ya-Xiang Yuan. Optimization Theory and Method: Nonlinear Programming. Optimization and its Applications. Springer Science +Business Media. LLC. USA. 2006.

7. Zhang Jianzhong, $\mathrm{Xu}$ Chengxian. Properties and Numerical Performance of Modified Quasi -Newton Methods Equations. J. Compt. Appl. Math. 2001; 137: 269-278.

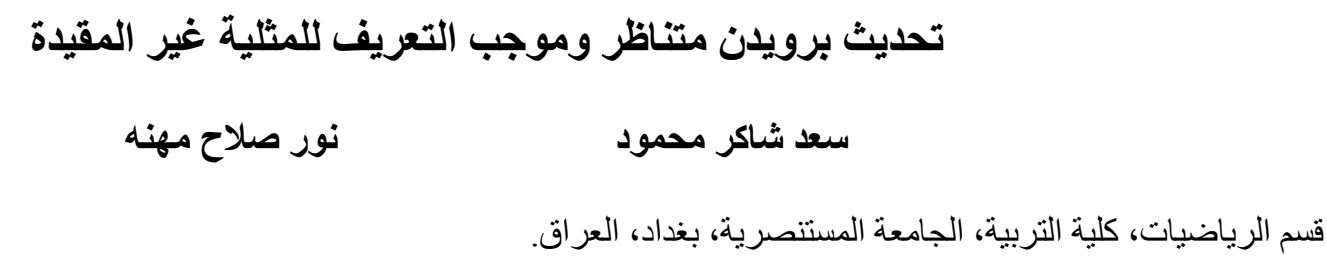

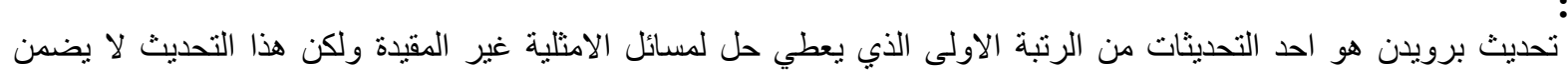

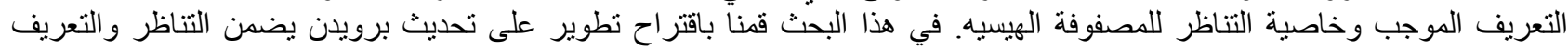
الموجب للمصفوفة الهيسيه وذلك بتحديث المتجه مستمرة وقابله للاشتقاق مرتين. قمنا بتقديم تقرير حول النتائج العددية حيث اجنئ النرينا مقارنة بين الطريقة المقترحة و طريقة برويدن لمسائل قياسية. الكلمات المفتاحية: تحديث برويدن، مصفوفة هيسين، شرط شبه نيونن 